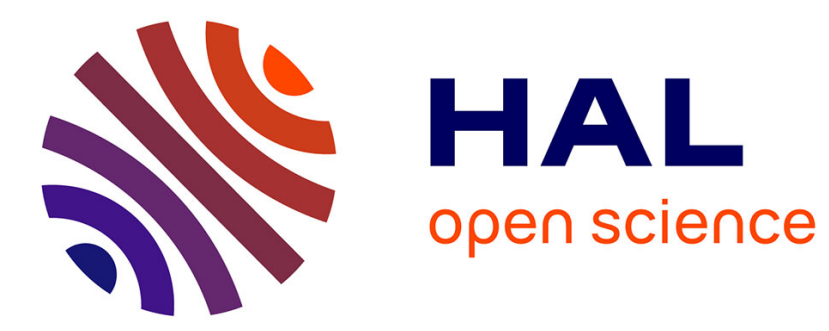

\title{
A Zone of Quiet Based Approach to Integrated Active Noise Control and Noise Reduction in Hearing Aids
}

\author{
Romain Serizel, Marc Moonen, Jan Wouters, Søren Holdt Jensen
}

\section{To cite this version:}

Romain Serizel, Marc Moonen, Jan Wouters, Søren Holdt Jensen. A Zone of Quiet Based Approach to Integrated Active Noise Control and Noise Reduction in Hearing Aids. IEEE Workshop on Applications of Signal Processing to Audio and Acoustics (WASPAA), Oct 2009, New Paltz, United States. hal-01393983

\section{HAL Id: hal-01393983 \\ https://hal.science/hal-01393983}

Submitted on 8 Nov 2016

HAL is a multi-disciplinary open access archive for the deposit and dissemination of scientific research documents, whether they are published or not. The documents may come from teaching and research institutions in France or abroad, or from public or private research centers.
L'archive ouverte pluridisciplinaire HAL, est destinée au dépôt et à la diffusion de documents scientifiques de niveau recherche, publiés ou non, émanant des établissements d'enseignement et de recherche français ou étrangers, des laboratoires publics ou privés. 


\title{
A Zone of Quiet Based Approach to Integrated Active Noise Control and Noise Reduction in Hearing Aids ${ }^{1}$
}

\author{
Romain Serizel ${ }^{2}$, Marc Moonen ${ }^{2}$, \\ Jan Wouters ${ }^{3}$ and Søren Holdt Jensen ${ }^{4}$
}

May 2009

In Proc. of the 2009 IEEE Workshop on Applications of Signal Processing to Audio and Acoustics (WASPAA 2009), New Paltz, U.S.A., Oct 2009

\footnotetext{
${ }^{1}$ This report is available by anonymous ftp from ftp.esat.kuleuven.be in the directory pub/sista/rserizel/reports/09-114.pdf

${ }^{2}$ K.U.Leuven, Dept. of Electrical Engineering (ESAT), Research group SCD (SISTA) Kasteelpark Arenberg 10, 3001 Leuven, Belgium, Tel. +32 1632 9607, Fax +32 16 321970, E-mail: romain.serizel@esat.kuleuven.be. This research work was carried out at the ESAT laboratory of the Katholieke Universiteit Leuven, in the frame of the Marie-Curie Fellowship EST-SIGNAL program (http://est-signal.i3s.unice.fr) under contract No. MEST-CT-2005-021175, and the Concerted Research Action GOA-AMBioRICS. The scientific responsibility is assumed by its authors.

${ }^{3}$ Katholieke Universiteit Leuven, Department of Neurosciences, ExpORL, O. \& N2, Herestraat 49/721, 3000 Leuven, Belgium, E-mail: Jan.Wouters@med.kuleuven.be

${ }^{4}$ Aalborg University, Department of Electronic Systems, MISP, Niels Jernes Vej 12 A6-3, 9220 Aalborg, Denmark, E-mail: shj@es.aau.dk
} 


\begin{abstract}
This paper presents an integrated approach to active noise control and noise reduction in hearing aids which is based on an optimization over a zone of quiet generated by the active noise control. A basic integrated scheme has been introduced previously to tackle secondary path effects and effects of noise leakage through an open fitting. This scheme, however, only takes the sound pressure at the ear canal microphone into account. In practice, it is desired to achieve noise control in a zone not limited to a single point. A scheme based on an average mean squared error criterion over the desired zone of quiet is presented here and compared experimentally with the original scheme.
\end{abstract}




\title{
A ZONE OF QUIET BASED APPROACH TO INTEGRATED ACTIVE NOISE CONTROL AND NOISE REDUCTION IN HEARING AIDS
}

\author{
Romain Serizel $^{1}$, Marc Moonen ${ }^{1}$, Jan Wouters ${ }^{2}$ and Søren Holdt Jensen ${ }^{3}$ \\ ${ }^{1}$ Katholieke Universiteit Leuven, ESAT-SCD, Kasteelpark Arenberg 10, B-3001 Leuven, Belgium \\ ${ }^{2}$ Katholieke Universiteit Leuven, ExpORL, O.\& N2, Herestraat 49/721, B-3000 Leuven, Belgium \\ ${ }^{3}$ Aalborg University, Dept. Electronic Systems, Niels Jernes Vej 12, DK-9220 Aalborg, Denmark \\ romain.serizel@esat.kuleuven.be
}

\begin{abstract}
This paper presents an integrated approach to active noise control and noise reduction in hearing aids which is based on an optimization over a zone of quiet generated by the active noise control. A basic integrated scheme has been introduced previously to tackle secondary path effects and effects of noise leakage through an open fitting. This scheme, however, only takes the sound pressure at the ear canal microphone into account. In practice, it is desired to achieve noise control in a zone not limited to a single point. A scheme based on an average mean squared error criterion over the desired zone of quiet is presented here and compared experimentally with the original scheme.
\end{abstract}

Index Terms - Active noise control, zone of quiet, multichannel wiener filter, noise reduction, hearing aids

\section{INTRODUCTION}

The usage of hearing aids with an open fitting has become more common over the past years mainly owing to the availability of more efficient feedback control schemes and fast signal processing units. Whereas removing the earmold reduces the occlusion effect and improves the physical comfort [1], one major drawback is that the noise leakage through the fitting cannot be neglected anymore.

One efficient way to cancel this undesired noise leakage is to use Active Noise Control (ANC) [2][3]. In the hearing aids framework, ANC then has to be performed together with a Noise Reduction (NR) scheme [4][5]. A scheme integrating the two functional blocks and based on a filtered-x [6][7][8] version of the Multichannel Wiener Filter (MWF) scheme (the so-called FxMWF) has been introduced in [9][10]. The objectives of this scheme are to attenuate the noise component of the leakage (i.e. ANC) and to minimize the difference between an unkown desired speech signal and the signal delivered at the tympanic membrane (i.e. NR). In pratice however, the so-called ear canal microphone, used to construct the error signal in the scheme, cannot be located exactly at the tympanic membrane. Besides, the spatial distribution of sound sources and the geometry of the ear canal do not allow to achieve control over the complete ear canal. Therefore, the actual signal-to-noise ratio (SNR) at the tympanic membrane is basically unkown and uncontrolled.

This research work was carried out at the ESAT Laboratory of Katholieke Universiteit Leuven, in the frame of the Marie-Curie Fellowship EST-SIGNAL (http://est-signal.i3s.unice.fr) under contract No. MEST-CT-2005-021175, and the Concerted Research Action GOAAMBioRICS. The scientific responsibility is assumed by its authors.
ANC allows to generate zones of quiet based on destructive interference. The size and shape of these zones of quiet depend on the type of the sound sources and the frequency components of the signal to be cancelled [11][12][13]. It is therefore possible to determine the performance of the scheme described in [10] at any point of the ear canal, based on the spatial distribution of the sound sources and on the position of the ear canal microphone.

In a similar way, it is also possible to compute an average of a particular design criterion, e.g., a mean squared error (MSE) criterion, over the desired zone of quiet. In the approach presented here a filter is derived from the minimization of this averaged-MSE (aMSE) criterion in order to achieve a control that is more robust on the desired zone of quiet than with a scheme minimizing a standard MSE criterion as in [10].

This paper will present a perfomance comparison between the original integrated ANC and NR scheme and the scheme introduced here, adjusted to a specific zone of quiet, both of them based on FxMWF and applied in hearing aids with an open fitting. The signal model, the integrated ANC and NR scheme and its performance on a defined spatial zone are described in Section 2. Section 3 introduces the approach based on a zone of quiet for integrated ANC and NR. Experimental results are presented in Section 4 and finally Section 5 presents a summary of the paper.

\section{BACKGROUND AND PROBLEM STATEMENT}

Speech enhancement in hearing aids is based on standard NR techniques ignoring the effects of noise leakage through the fitting and the secondary path between the loudspeaker and the tympanic membrane. The leakage signal is not processed in the hearing aid therefore it is not possible to improve its SNR using standard NR schemes. It has been shown in [10] that leakage signal can degrade NR performance for amplification gain up to $20 \mathrm{~dB}$. It is possible however to attenuate the leakage signal's noise component using ANC. This section introduces the signal model and notation, suggests a frequency-domain version of the integrated scheme presented in [9][10] and introduces the model to compute the SNR over the so-called zone of quiet.

\subsection{Signal model}

Let $M$ be the number of microphones (channels). The frequencydomain signal $X_{m}$ for microphone $m$ has a desired speech part $X_{m}^{s}$ and an additive noise part $X_{m}^{n}$, i.e.:

$$
X_{m}(\omega)=X_{m}^{s}(\omega)+X_{m}^{n}(\omega) \quad m \in\{1 \ldots M\}
$$


where $\omega=2 \pi f$ is the frequency-domain variable.

In the sequel, superscripts $s$ and $n$ will also be used for other signals and vectors, to denote their speech and noise component, respectively. Signal model (1) holds for so-called "speech plus noise periods". There are also "noise only periods" (i.e. speech pauses), during which only a noise component is observed. The compound vector gathering all channels is:

$$
\mathbf{X}^{T}(\omega)=\left[X_{1}(\omega) \ldots X_{M}(\omega)\right]
$$

An optimal (Wiener) filter $\mathbf{W}^{T}(\omega)=\left[W_{1}(\omega) \ldots W_{M}(\omega)\right]$ will be designed and applied to the signals, which minimizes a Mean Squared Error (MSE) criterion:

$$
J_{M S E}(\omega)=\mathbb{E}\left\{|E(\omega)|^{2}\right\}
$$

Here $E(\omega)$ is an error signal constructed from the ear canal microphone signal.

The filter output signal $Z(\omega)$ is defined as:

$$
Z(\omega)=\mathbf{W}^{H}(\omega) \mathbf{X}(\omega)
$$

which will be the hearing aid output signal, fed to the loudspeaker.

\subsection{Integrated active noise control and noise reduction}

This subsection presents a frequency-domain version of the scheme introduced in [9][10] which integrates both the NR and an ANC in a single set of adaptive filters and which is derived as follows.

The so-called secondary path represents the propagation from the loudspeaker to the tympanic membrane (including the loudspeaker response itself). As in any ANC scheme, it has to be taken into account explicitly. Assuming that the loudspeaker characteristic is approximately linear, the secondary path can be represented by the transfer function $C(\omega)$.

The purpose of the NR is to provide an optimal estimate of a desired signal $D_{N R}(\omega)$, which is chosen to be equal to the (unknown) speech component in the first microphone, up to a delay $\Delta$ and amplified by a gain $G$, representing the hearing loss compensation:

$$
D_{N R}(\omega)=G \cdot X_{1}^{s}(\omega) \mathrm{e}^{-j \omega \Delta}
$$

The aim is to deliver this desired signal at the tympanic membrane in spite of the secondary path.

Finally, the purpose of the ANC is to cancel the noise component $L^{n}(\omega)$ of the leakage signal $L(\omega)$ arriving at the tympanic membrane through the open fitting. In the hearing aids context, the speech component of the leakage signal can provide cues which, e.g., are helpful for speaker localization. Therefore, it is chosen here to cancel only the noise component of the leakage signal and preserve its speech component.

The overall desired signal (at the tympanic membrane) to be used is then:

$$
D_{\text {Int }}(\omega)=-L^{n}(\omega)+D_{N R}(\omega)
$$

Hence the MSE criterion to be minimized is:

$$
\begin{aligned}
J_{M S E}(\omega) & =\mathbb{E}\left\{\left|E_{\text {Int }}(\omega)\right|^{2}\right\} \\
E_{\text {Int }}(\omega) & =C(\omega) \cdot \mathbf{W}^{H}(\omega) \mathbf{X}(\omega)+L^{n}(\omega)-D_{N R}(\omega)
\end{aligned}
$$

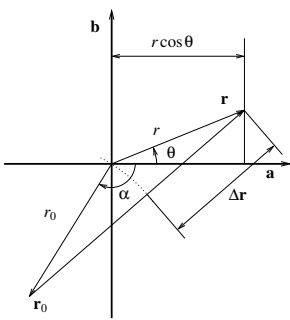

a) Secondary source

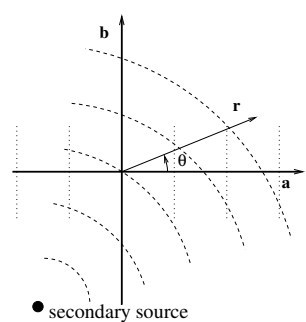

b) Sound field
Figure 1: Sources distribution and sound field. The dashed circles represent the sound from the secondary source, the dotted lines, the sound from the leakage.

The scheme relies on a filtered-x type operation based on an estimate $\hat{C}(\omega)$ of the secondary path $C(\omega)$. The filtered reference signals are:

$$
\begin{aligned}
Y_{m}(\omega) & =\hat{C}(\omega) X_{m}(\omega) \quad m \in\{1 \ldots M\} \\
\mathbf{Y}^{T}(\omega) & =\left[Y_{1}(\omega) \ldots Y_{M}(\omega)\right]
\end{aligned}
$$

Assuming that the secondary path identification error is small $(\hat{C}(\omega) \approx C(\omega))$ and that the filter $\mathbf{W}$ is adapting slowly, the MSE criterion (7) can be written as follows:

$$
J_{M S E}(\omega) \approx \mathbb{E}\left\{\left|\mathbf{W}^{H}(\omega) \mathbf{Y}(\omega)+L^{n}(\omega)-D_{N R}(\omega)\right|^{2}\right\}
$$

The optimal filter (FxMWF) minimizing (7) is then:

$$
\mathbf{W}_{\text {Int }}(\omega)=\mathbf{R}_{Y Y}^{-1}(\omega) \mathbf{r}_{Y D_{I n t}}(\omega)
$$

Here $\mathbf{R}_{Y Y}(\omega)$ is the correlation matrix of the filtered reference signal $\mathbf{Y}(\omega)$ and $\mathbf{r}_{Y D_{\text {Int }}}(\omega)$ is the cross-correlation vector between the filtered reference signal $\mathbf{Y}(\omega)$ and the desired signal $D_{\text {Int }}(\omega)$.

The time-domain version of the above scheme, based on an adaptation employing an error signal (7) measured with an ear canal microphone, has been derived and evaluated in [9][10]. Note that in practice, to ensure the causality of the frequency-domain version of the scheme, the filter coefficients are computed in the frequency-domain while filtering operation is in the time-domain, in a similar way as presented in [14].

\subsection{Zone of quiet}

The scheme described above achieves noise control at the ear canal microphone. Ideally, it should be located at the tympanic membrane. In pratice, however, this is not possible, i.e., the controlled point can be located a few tens of millimeters away. The ANC can then only generate a so-called zone of quiet around the ear canal microphone and the signal which is actually reaching the tympanic membrane is unkown and uncontrolled.

To study the performance of the scheme over the zone of quiet, the ear canal microphone is chosen to be the origin of the 2-dimensional coordinate system used to determine each point of the space (presented in fig. 1-a). It is possible then to estimate the sound pressure at a particular point $(\mathbf{r}=(r \sin \theta, r \cos \theta))$ of the ear canal, when the sound pressure at the ear canal microphone and the type of the sound sources generating the sound field in the ear canal are known [11][12][13]. 
The external sound sources (the speaker and the noise sources) are assumed to be far enough from the hearing aid user so that the leakage signal can be viewed as a far field signal and so a plane wave model can be used. Assuming also that the ear canal acts as a waveguide and that the plane waves from different directions are uncorrelated [15], the sound field resulting from the leakage can be modeled as one plane wave propagating along the ear canal axis (chosen to be the axis a) as presented on fig. 1-b. The sound pressure at point $\mathbf{r}$, resulting from the leakage signal can then be expressed as follows:

$$
\begin{aligned}
& L(\omega, \mathbf{r})=L(\omega, \mathbf{0}) \mathrm{e}^{-j \omega \frac{r \cos \theta}{c_{0}}} \\
& \delta_{1}(\omega, \mathbf{r}) \triangleq \mathrm{e}^{-j \omega \frac{r \cos \theta}{c_{0}}}
\end{aligned}
$$

where $c_{0}$ is the speed of sound in air.

The hearing aid loudspeaker (secondary source), is located at $\mathbf{r}_{0}=\left(r_{0} \sin \alpha, r_{0} \cos \alpha\right)$. The ear canal is assumed to be in the near field of the hearing aid loudspeaker. A simple model to study the sound field generated by the hearing aid loudspeaker is then the monopole point secondary source approach [12]. The use of this model can be justified by the small dimension of the hearing aid loudspeaker. The sound field generated by the hearing aid loudspeaker is then assumed to be circular with decaying amplitude:

$$
\begin{aligned}
& U(\omega, \mathbf{r})=U(\omega, \mathbf{0}) \frac{r_{0}}{\left|\mathbf{r}-\mathbf{r}_{0}\right|} \mathrm{e}^{-j \omega \frac{\Delta \mathbf{r}}{c_{0}}} \\
& \delta_{2}(\omega, \mathbf{r}) \triangleq \frac{r_{0}}{\left|\mathbf{r}-\mathbf{r}_{0}\right|} \mathrm{e}^{-j \omega \frac{\Delta \mathbf{r}}{c_{0}}}
\end{aligned}
$$

Using Parseval theorem, the power of the total sound pressure signal and the SNR at the point $\mathbf{r}$ can be expressed as follows:

$$
\begin{aligned}
\operatorname{Pow}(\mathbf{r}) & =\frac{1}{2 \pi} \int_{-\infty}^{\infty}\left|\delta_{1}(\omega, \mathbf{r}) L(\omega)+\delta_{2}(\omega, \mathbf{r}) U(\omega)\right|^{2} \mathrm{~d} \omega \\
\operatorname{SNR}(\mathbf{r}) & =\frac{\operatorname{Pow}^{s}(\mathbf{r})}{\operatorname{Pow}^{n}(\mathbf{r})}
\end{aligned}
$$

The filter $\mathbf{W}_{\text {Int }}$ introduced in the previous section is optimal only at the ear canal microphone. A different filter $\mathbf{W}_{\text {Int }}(\mathbf{r})$ may be defined corresponding to each point $\mathbf{r}$. This filter then minimizes the MSE criterion (7) rewritten at point $\mathbf{r}$, based on (11) and (13):

$$
J_{M S E}(\omega, \mathbf{r})=\mathbb{E}\left\{\left|U(\omega, \mathbf{r})+L^{n}(\omega, \mathbf{r})-D_{N R}(\omega)\right|^{2}\right\}
$$

It appears clearly that the SNR as defined in (15) cannot be a satisfying measure if the performance of the scheme is to be studied on a spatial zone. Let us therefore define the average SNR over the desired zone (area $S$ ) as:

$$
a S N R \triangleq \frac{1}{S} \int_{S} S N R(\mathbf{r}) \mathrm{d} S
$$

The filter $\mathbf{W}_{\text {Int }}$ introduced in the previous section minimizes the MSE criterion (7) at the ear canal microphone and may exhibit degraded performance in terms of average SNR (see also Section 4).

\section{INTEGRATED ACTIVE NOISE CONTROL AND NOISE REDUCTION BASED ON A ZONE OF QUIET}

It has been explained in the previous section how the sound pressure and the SNR are varying when moving away from the ear canal microphone. Therefore, the average-SNR measure (17) has been introduced. In order to derive a filter $\mathbf{W}_{\text {avg }}$, integrating both the NR and an ANC, which is efficient on a desired zone (i.e., in term of the average-SNR), the aMSE has to be defined:

$$
a J_{M S E}(\omega)=\frac{1}{S} \int_{S} J_{M S E}(\omega, \mathbf{r}) \mathrm{d} S
$$

Assuming that the secondary path identification error is small $(\hat{C}(\omega) \approx C(\omega))$, that the filter $\mathbf{W}$ is adapting slowly and that the noise and speech components are uncorrelated, the MSE criterion (16) can be written as follows:

$$
\begin{array}{r}
J_{M S E}(\omega, \mathbf{r}) \approx \mathbb{E}\left\{\left|\delta_{2}(\omega, \mathbf{r}) \mathbf{W}^{H}(\omega) \mathbf{Y}^{s}(\omega)-D_{N R}(\omega)\right|^{2}\right\} \\
+\mathbb{E}\left\{\left|\delta_{2}(\omega, \mathbf{r}) \mathbf{W}^{H}(\omega) \mathbf{Y}^{n}(\omega)+\delta_{1}(\omega, \mathbf{r}) L^{n}(\omega)\right|^{2}\right\}
\end{array}
$$

The optimal filter (FxMWF) minimizing (18) is then:

$$
\mathbf{W}_{a v g}(\omega)=\mathbf{R}_{\text {avg }}(\omega)^{-1} \mathbf{r}_{a v g}(\omega)
$$

where

$$
\begin{aligned}
\mathbf{R}_{a v g}(\omega) & =\eta_{y}(\omega) \mathbf{R}_{Y Y}(\omega) \\
\mathbf{r}_{a v g}(\omega) & =\eta_{s}(\omega) \mathbf{r}_{Y^{s} D_{N R}}(\omega)-\eta_{n}(\omega) \mathbf{r}_{Y^{n} L^{n}}(\omega)
\end{aligned}
$$

with

$$
\begin{aligned}
& \eta_{y}(\omega) \triangleq \frac{1}{S} \int_{S}\left|\delta_{2}(\omega, \mathbf{r})\right|^{2} \mathrm{~d} S \\
& \eta_{s}(\omega) \triangleq \frac{1}{S} \int_{S} \delta_{2}(\omega, \mathbf{r}) \mathrm{d} S \\
& \eta_{n}(\omega) \triangleq \frac{1}{S} \int_{S} \delta_{2}(\omega, \mathbf{r}) \delta_{1}(\omega, \mathbf{r})^{*} \mathrm{~d} S
\end{aligned}
$$

Here $\mathbf{R}_{Y Y}(\omega)$ is the correlation matrix of the filtered reference signal $\mathbf{Y}(\omega)$, while $\mathbf{r}_{Y^{s} D_{N R}}(\omega)$ and $\mathbf{r}_{Y^{n} L^{n}}(\omega)$ are the crosscorrelation vectors between the filtered reference signal $\mathbf{Y}(\omega)$ and, the desired speech signal $D_{N R}(\omega)$ and the noise component of the leakage signal $L^{n}(\omega)$, respectively.

\section{EXPERIMENTAL RESULTS}

The scheme introduced in Section 3 has been tested experimentally and its performance has been compared with the performance of the frequency-domain version of the standard integrated scheme [9][10] described in Section 2.2.

The simulations were run for a two-microphone behind-theear (BTE) hearing aid, with a speech source at $0^{\circ}$ and a babble noise source at $270^{\circ}$. The BTE is worn on the left ear, facing the noise source. The SNR for the source signals is set to $5 d B$ and the amplification gain $G=15 d B$.

In our case, as the two algorithms also perform ANC, the leakage SNR, which can also be considered as the SNR when the hearing aid is turned off, is taken as a reference. Note that it can be shown from (11) that the SNR of the leakage signal is stationary in space, i.e., in the ear canal. The intelligibility-weighted signal-tonoise ratio improvement [16] is used here to compute the averageSNR improvement which is defined as

$$
\begin{aligned}
a \Delta S N R_{\text {intellig }} & =\frac{1}{S} \int_{S} \Delta S N R_{\text {intellig }}(\mathbf{r}) \mathrm{d} S \\
\Delta S N R_{\text {intellig }}(\mathbf{r}) & =\sum_{i} I_{i}\left(S N R_{i, \text { out }}(\mathbf{r})-S N R_{i, \text { leak }}\right)
\end{aligned}
$$




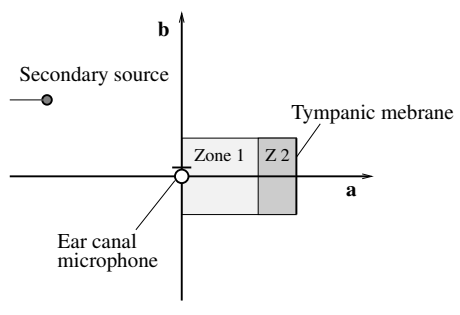

Figure 2: Simulation setup.

\begin{tabular}{|l|c|c|}
\hline & $\min (\mathrm{SNR})$ & aSNR \\
\hline \hline Unconstrained integrated & 6.5 & 8 \\
\hline Adjusted integrated (Zone 1) & 7.8 & 8.9 \\
\hline
\end{tabular}

Table 1: Minimum and average SNR, over zone 1 (in $d B$ ).

where $I_{i}$ is the band importance function and $\operatorname{SNR}_{i, \text { out }}(\mathbf{r})$ and $\mathrm{SNR}_{i, \text { leak }}$ represent the output $\mathrm{SNR}$ at point $\mathbf{r}$ and the leakage SNR (in $\mathrm{dB}$ ) of the $i$ th band, respectively.

In these experiments, the ear canal microphone was considered to be located $15 \mathrm{~mm}$ away from the tympanic membrane. The hearing aid loudspeaker is located $20 \mathrm{~mm}$ away from ear canal microphone and the angle $\alpha$ is set to $\frac{5 \pi}{6}$ (as presented on fig. 2). Two different zones are considered here, the first zone (zone 1 on fig. 2) is the portion of the ear canal between the ear canal microphone and the tympanic membrane (it is assumed to be a $15 \mathrm{~mm}$ by $10 \mathrm{~mm}$ rectangular zone), the second zone (zone 2 on fig. 2) is the close neighbourhood of the tympanic membrane (considered to be a $5 \mathrm{~mm}$ by $10 \mathrm{~mm}$ rectangular zone). The average-SNR and the minimum SNR have been computed over the two zones for the output of the standard integrated ANC and NR and the scheme presented in Section 3, adjusted to the corresponding zones. The results are shown in table 1 for the ear canal (zone 1) and in table 2 for the neighbourhood of the tympanic membrane (zone 2).

In both cases, the scheme presented here, adjusted to the zone on which the noise is to be cancelled, exhibits improved performance in average-SNR compared to the standard integrated scheme. It also allows to improve the minimum SNR, therefore reducing the impact of the performance degradation where it is the most significant.

\section{CONCLUSION}

This paper presented an integrated approach to ANC and NR in hearing aids based on an optimization over a zone of quiet generated by the ANC. While the standard integrated scheme only allows to achieve control at the ear canal microphone, which is in pratice away from the tympanic membrane, the scheme proposed here allows to control the noise in a desired zone. Therefore, it exhibits improved performance, compared against the standard scheme, especially when the noise is to be cancelled on an zone which is distant from the ear canal microphone.

\section{REFERENCES}

[1] J. Kiessling, "Sounds towards the tympanic membrane," in 8th EFAS Congress. Heidelberg: European Federation of

\begin{tabular}{|l|c|c|}
\hline & $\min ($ SNR $)$ & aSNR \\
\hline \hline Unconstrained integrated & 6.5 & 7.2 \\
\hline Adjusted integrated (Zone 2) & 8.8 & 9.2 \\
\hline
\end{tabular}

Table 2: Minimum and average SNR, over zone 2 (in $d B$ ).

Audiological Societies, June 2007.

[2] S. Elliott and P. Nelson, active control of sound. Cambridge: Academic press, 1993.

[3] S. Kuo and D. Morgan, "Active noise control: a tutorial review," Proceedings of the IEEE, vol. 87, Issue: 6, no. 00189219, pp. 943- 973, Jun 1999.

[4] S. Doclo, A. Spriet, J. Wouters, and M. Moonen, "Frequency-domain criterion for the speech distortion weighted multichannel wiener filter for robust noise reduction," Speech Communication, vol. 49, no. 7-8, pp. 636-656, 2007.

[5] L. Griffiths and C. Jim, "An alternative approach to linearly constrained adaptive beamforming," Antennas and Propagation, IEEE Transactions on, vol. 30, pp. 27-34, 1982.

[6] E. Bjarnason, "Analysis of the filtered-x lms algorithm," Speech and Audio Processing, IEEE Transactions on, vol. 3, no. 6, pp. 504-514, 1995.

[7] J. C. Burgess, "Active adaptive sound control in a duct: a computer simulation," The Journal of the Acoustical Society of America, vol. 70, Issue 3, pp. 715-726 715-726, September 1981.

[8] B. Widrow and S. Stearns, Adaptive signal processing. EnglewoodC liffs,N J: Prentice-Hall, 1985.

[9] R. Serizel, M. Moonen, J. Wouters, and S. H. Jensen, "Combined active noise control and noise reduction in hearing aids," in 11th International Workshop on Acoustic Echo and Noise Control (IWAENC), September 2008.

[10] — , "Integrated active noise control and noise reduction in hearing aids," ESAT-SISTA, K.U.Leuven submitted for publication, (Leuven, Belgium), Tech. Rep., 2009.

[11] P. Nelson, J. Hammond, P. Joseph, and S. Elliott, "Active control of stationary random sound fields," The Journal of the Acoustical Society of America, vol. 87, p. 963, 1990.

[12] P. Joseph, S. J. Elliott, and P. A. Nelson, "Near field zones of quiet," Journal of Sound and Vibration, vol. 172, issue 5, pp. 605-627, may 1994.

[13] B. Rafaely, "Zones of quiet in a broadband diffuse sound field," The Journal of the Acoustical Society of America, vol. 110, p. 296, 2001.

[14] D. Morgan and J. Thi, "A delayless subband adaptive filter architecture," Signal Processing, IEEE Transactions on, vol. 43, no. 8, pp. 1819-1830, 1995.

[15] B. Rafaely, "Spatial-temporal correlation of a diffuse sound field," The Journal of the Acoustical Society of America, vol. 107, pp. 3254-3258, 2000.

[16] J. Greenberg, P. Peterson, and P. Zurek, "Intelligibilityweighted measures of speech-to-interference ratio and speech system performance," The Journal of the Acoustical Society of America, vol. 94, no. 5, pp. 3009-3010, Nov. 1993. 